CRYSTALLOGRAPHIC COMMUNICATIONS

ISSN 2056-9890

Received 1 November 2017

Accepted 3 November 2017

Edited by A. J. Lough, University of Toronto, Canada

Keywords: crystal structure; chloranilic acid; 2carboxypyridine; 2-carboxyquinoline; twitterion; disorder; hydrogen bond.

CCDC references: $1583721 ; 1583720$

Supporting information: this article has supporting information at journals.iucr.org/e

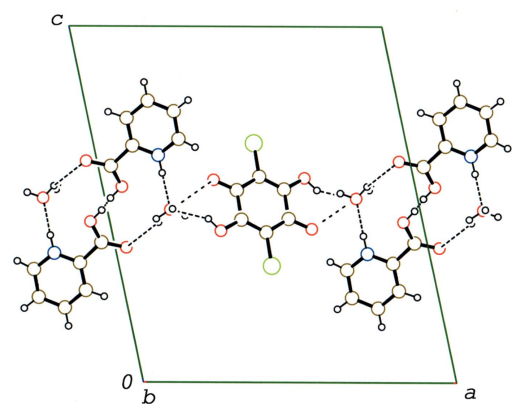

OPEN $\odot$ ACCESS

\section{Crystal structures of two 1:2 dihydrate compounds of chloranilic acid with 2-carboxypyridine and 2-carboxyquinoline}

\author{
Kazuma Gotoh and Hiroyuki Ishida*
}

Department of Chemistry, Faculty of Science, Okayama University, Okayama 700-8530, Japan. *Correspondence e-mail: ishidah@cc.okayama-u.ac.jp

The crystal structure of the 1:2 dihydrate compound of chloranilic acid (systematic name: 2,5-dichloro-3,6-dihydroxy-1,4-benzoquinone) with 2-carboxypyridine (another common name: picolinic acid; systematic name: pyridine2-carboxylic acid), namely, $2 \mathrm{C}_{6} \mathrm{H}_{5.5} \mathrm{NO}_{2}{ }^{0.5+} \cdot \mathrm{C}_{6} \mathrm{HCl}_{2} \mathrm{O}_{4}{ }^{-} \cdot 2 \mathrm{H}_{2} \mathrm{O}$, (I), has been determined at $180 \mathrm{~K}$, and the structure of the 1:2 dihydrate compound of chloranilic acid with 2-carboxyquinoline (another common name: quinaldic acid; systematic name: quinoline-2-carboxylic acid), namely, $2 \mathrm{C}_{10} \mathrm{H}_{7} \mathrm{NO}_{2} \cdot \mathrm{C}_{6} \mathrm{H}_{2}$ $\mathrm{Cl}_{2} \mathrm{O}_{4} \cdot 2 \mathrm{H}_{2} \mathrm{O}$, (II), has been redetermined at $200 \mathrm{~K}$. This determination presents a higher precision crystal structure than the previously published structure [Marfo-Owusu \& Thompson (2014). X-ray Struct. Anal. Online, 30, 55-56]. Compound (I) was analysed as a disordered structure over two states, viz. salt and co-crystal. The salt is bis(2-carboxypyridinium) chloranilate dihydrate, $2 \mathrm{C}_{6} \mathrm{H}_{6} \mathrm{NO}_{2}{ }^{+} \cdot \mathrm{C}_{6} \mathrm{Cl}_{2} \mathrm{O}_{4}{ }^{2-} \cdot 2 \mathrm{H}_{2} \mathrm{O}$, and the co-crystal is bis(pyridinium-2-carboxylate) chloranilic acid dihydrate, $2 \mathrm{C}_{6} \mathrm{H}_{5} \mathrm{NO}_{2} \cdot \mathrm{C}_{6} \mathrm{H}_{2} \mathrm{Cl}_{2} \mathrm{O}_{4} \cdot 2 \mathrm{H}_{2} \mathrm{O}$, including zwitterionic 2-carboxypyridine. In both salt and co-crystal, the water molecule links the chloranilic acid and 2-carboxypyridine molecules through $\mathrm{O}-\mathrm{H} \cdots \mathrm{O}$ and $\mathrm{N}-$ $\mathrm{H}$. . O hydrogen bonds. The 2-carboxypyridine molecules are connected into a head-to-head inversion dimer by a short $\mathrm{O}-\mathrm{H}$. . O hydrogen bond, in which the $\mathrm{H}$ atom is disordered over two positions. Compound (II) is a 1:2 dihydrate cocrystal of chloranilic acid and zwitterionic 2-carboxyquinoline. The water molecule links the chloranilic acid and 2-carboxyquinoline molecules through $\mathrm{O}-\mathrm{H}$... O hydrogen bonds. The 2-carboxyquinoline molecules are connected into a head-to-tail inversion dimer by a pair of $\mathrm{N}-\mathrm{H} \cdots \mathrm{O}$ hydrogen bonds.

\section{Chemical context}

Chloranilic acid, a dibasic acid with hydrogen-bond donor as well as acceptor groups, appears particularly attractive as a template for generating tightly bound self-assemblies with various pyridine derivatives as well as being a model compound for investigating hydrogen-transfer motions in $\mathrm{O}-$ $\mathrm{H} \cdots \mathrm{N}$ and $\mathrm{N}-\mathrm{H} \cdots \mathrm{O}$ hydrogen-bond systems (Zaman et al., 2004; Molčanov \& Kojić-Prodić, 2010; Seliger et al., 2009; Asaji et al. 2010). Previously, we have prepared three 1:1 compounds of chloranilic acid with 2-, 3- and 4-carboxypyridine and analysed the crystal structures in order to extend our study on $D-\mathrm{H} \cdots A$ hydrogen bonding $(D=\mathrm{N}, \mathrm{O}$ or $\mathrm{C} ; A=\mathrm{N}, \mathrm{O}$ or $\mathrm{Cl})$ in chloranilic acid-substituted pyridine systems (Gotoh et al., 2006, 2009; Tabuchi et al., 2005). In the present study, we have prepared a 1:2 compound of chloranilic acid with 2-carboxypyridine and also redetermined the structure of a 1:2 compound of chloranilic acid with 2-carboxyquinoline with higher precision than previously reported structure [Marfo- 
Owusu \& Thompson, 2014; although the title and text in this reference refer to the $1: 1$ adduct of chloranilic acid with 2-carboxyqulinone, the reported structure is the $1: 2$ compound, the same as the present compound (II)]. The crystal structure of the anhydrous 1:2 compound of chloranilic acid with 2-carboxyquinoline was also reported by MarfoOwusu \& Thompson (2016).<smiles>O=C(O)c1ccccn1</smiles><smiles>O=C1C(=O)C(Cl)C(=O)C(Cl)=C1Cl</smiles>
$2 \mathrm{H}_{2} \mathrm{O}$<smiles>O=C([O-])c1ccccn1</smiles><smiles>C=CC=C</smiles><smiles>O=C1C(O)=C(Cl)C(=O)C(O)=C1Cl</smiles>

$2 \mathrm{H}_{2} \mathrm{O}$

(I)<smiles>O=C1C(O)=C(Cl)C(=O)C(O)=C1Cl</smiles>
$2 \mathrm{H}_{2} \mathrm{O}$

(II)

\section{Structural commentary}

Compound (I) (Fig. 1) crystallizes with one-half of a chloranilic acid molecule, which is located on an inversion centre, one 2-carboxypyridine molecule and one water molecule in the asymmetric unit. In the crystal, the water molecule is disordered over two sites with equal occupancies of 0.5 . The occupancies of the $\mathrm{H}$ atoms in the chloranilic acid molecule and the carboxy group of the 2-carboxypyridine molecule are also 0.5. The compound is, therefore, considered to be a disordered state over two forms, viz. bis(2-carboxypyridinium) chloranilate dihydrate, $(A)$, and bis(pyridinium-2-carboxylate) chloranilic acid dihydrate, $(B)$, as shown in the scheme and Fig. 2. In form $(A)$, the water molecule acts as one $\mathrm{N}-\mathrm{H} \cdots \mathrm{O}$ hydrogen-bond acceptor and two $\mathrm{O}-\mathrm{H} \cdots \mathrm{O}$ hydrogen-bond donors $\left(\mathrm{N} 1-\mathrm{H} 1 \cdots \mathrm{O} 5 A, \mathrm{O} 5 A-\mathrm{O} 9 A \cdots \mathrm{O} 4^{\mathrm{ii}}\right.$ and $\mathrm{O} 5 A-$ $\mathrm{H} 10 A \cdots \mathrm{O} 2$; symmetry code as in Table 1$)$, while in form $(B)$, the water molecule acts as the acceptor of $\mathrm{N}-\mathrm{H} \cdots \mathrm{O}$ and $\mathrm{O}-$ $\mathrm{H} \cdots \mathrm{O}$ hydrogen bonds, and as two $\mathrm{O}-\mathrm{H} \cdots \mathrm{O}$ hydrogen-bond donors $\left(\mathrm{N} 1-\mathrm{H} 1 \cdots \mathrm{O} 5 B, \mathrm{O} 2-\mathrm{H} 2 \cdots \mathrm{O} 5 B, \mathrm{O} 5 B-\mathrm{H} 9 B \cdots \mathrm{O} 4^{\mathrm{ii}}\right.$ and $\mathrm{O} 5 B-\mathrm{H} 10 B \cdots \mathrm{O} 1^{\mathrm{iii}}$; Table 1). The dihedral angle
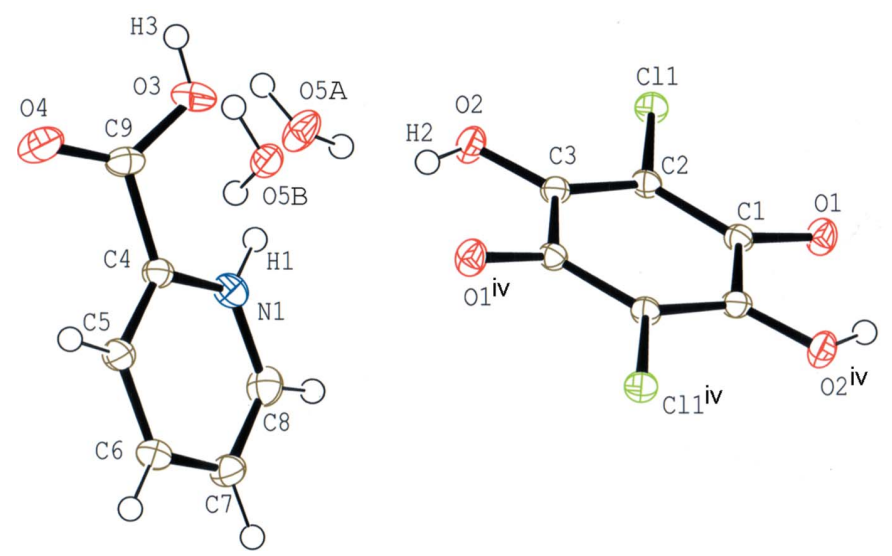

Figure 1

The molecular structure of compound (I), showing the atom-numbering scheme. Displacement ellipsoids of non-H atoms are drawn at the $50 \%$ probability level and $\mathrm{H}$ atoms are drawn as small spheres of arbitrary radii. The water molecule is disordered over two sites with equally occupancies. Atoms $\mathrm{H} 2$ and $\mathrm{H} 3$ have site-occupancy factors of 0.5 . [Symmetry code: (iv) $-x+1,-y+1,-z+1$.]

between the pyridine ring and the carboxy plane in the base molecule is $23.32(15)^{\circ}$.

The asymmetric unit of compound (II) consists of one-half of a chloranilic acid molecule, which is located on an inversion centre, one 2-carboxyquinoline molecule and one water molecule. In the crystal, the 2-carboxyquinoline molecule is in a
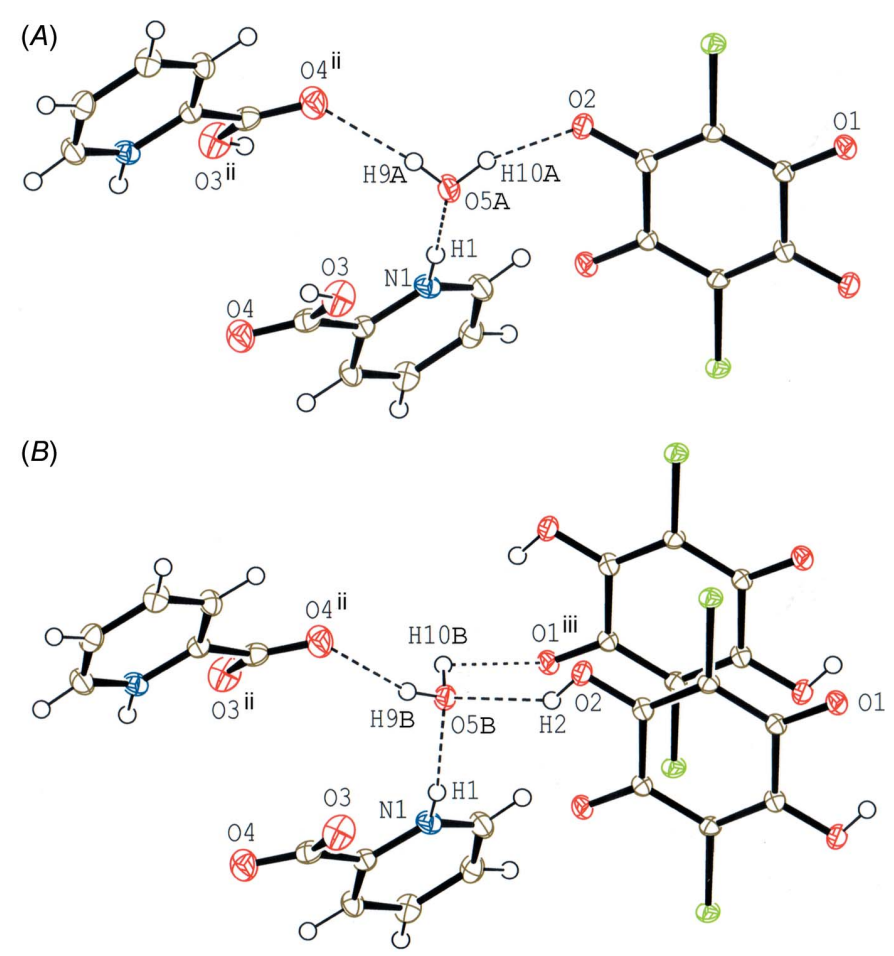

Figure 2

A partial packing diagram of compound (I) around the disordered water molecule in bis(2-carboxypyridinium) chloranilate dihydrate $(A)$ and bis(pyridinium-2-carboxylate) chloranilic acid dihydrate $(B)$, showing $\mathrm{O}-\mathrm{H} \cdots \mathrm{O}$ and $\mathrm{N}-\mathrm{H} \cdots \mathrm{O}$ hydrogen bonds (dashed lines). [Symmetry codes: (ii) $-x,-y,-z+1$; (iii) $-x+1,-y,-z+1$.] 
Table 1

Hydrogen-bond geometry $\left(\AA{ }^{\circ}\right)$ for $(\mathrm{I})$.

\begin{tabular}{lllll}
\hline$D-\mathrm{H} \cdots A$ & $D-\mathrm{H}$ & $\mathrm{H} \cdots A$ & $D \cdots A$ & $D-\mathrm{H} \cdots A$ \\
\hline $\mathrm{N} 1-\mathrm{H} 1 \cdots \mathrm{O} 5 A$ & $0.895(17)$ & $1.843(17)$ & $2.7380(19)$ & $179(2)$ \\
$\mathrm{N} 1-\mathrm{H} 1 \cdots \mathrm{O} 5 B$ & $0.895(17)$ & $1.907(17)$ & $2.7417(18)$ & $154(2)$ \\
$\mathrm{O} 2-\mathrm{H} 2 \cdots \mathrm{O} 5 B$ & $0.83(3)$ & $2.12(3)$ & $2.8111(19)$ & $141(3)$ \\
$\mathrm{O} 3-\mathrm{H} 3 \cdots 3^{\mathrm{i}}$ & $0.82(3)$ & $1.62(4)$ & $2.4352(14)$ & $174(4)$ \\
$\mathrm{O} 5 A-\mathrm{H} 9 A \cdots \mathrm{O} 4^{\mathrm{ii}}$ & $0.82(3)$ & $2.11(3)$ & $2.927(2)$ & $170(3)$ \\
$\mathrm{O} 5 B-\mathrm{H} 9 B \cdots \mathrm{O} 4^{\mathrm{ii}}$ & $0.82(3)$ & $2.02(3)$ & $2.8159(19)$ & $162(3)$ \\
$\mathrm{O}^{2} A-\mathrm{H} 10 A \cdots \mathrm{O} 2$ & $0.84(3)$ & $1.90(3)$ & $2.6762(19)$ & $154(4)$ \\
$\mathrm{O} 5 B-\mathrm{H} 10 B \cdots \mathrm{O} 1^{\mathrm{iii}}$ & $0.85(2)$ & $2.60(3)$ & $3.095(2)$ & $119(2)$ \\
$\mathrm{C} 8-\mathrm{H} 8 \cdots \mathrm{C} 11^{\text {iii }}$ & 0.95 & 2.78 & $3.6524(12)$ & 154 \\
$\mathrm{C} 8-\mathrm{H} 8 \cdots \mathrm{O} 1^{\mathrm{iii}}$ & 0.95 & 2.47 & $3.1871(14)$ & 132
\end{tabular}

Symmetry codes: (i) $\quad-x,-y+1,-z+1 ; \quad$ (ii) $\quad-x,-y,-z+1$; $\quad$ (iii)

$-x+1,-y,-z+1$.

twitterionic form and no acid-base interaction involving $\mathrm{H}$ atom transfer between chloranilic acid and 2-carboxyquinoline is observed (Fig. 3). The dihedral angle between the quinoline ring system and the carboxylate plane in the base molecule is $20.84(19)^{\circ}$. The water molecule acts as an $\mathrm{O}-$ $\mathrm{H}$... O hydrogen-bonding bridge between the chloranilic and 2-carboxyquinoline molecules $(\mathrm{O} 2-\mathrm{H} 2 \cdots \mathrm{O} 5$ and $\mathrm{O} 5-$ H3...O4; Table 2).

\section{Supramolecular features}

In the crystal of compound (I), the 2-carboxypyridine molecules, which are related by an inversion centre, are linked into a head-to-head dimer via a short $\mathrm{O}-\mathrm{H} \cdots \mathrm{O}$ hydrogen bond, in which the $\mathrm{H}$ atom is disordered over two sites (O3H3...O3 ${ }^{\mathrm{i}}$; Table 1), as observed in pyridinium-2-carboxylic acid pyridinium-2-carboxylate perchlorate (Wang et al., 2015). The three components are linked via the above-mentioned $\mathrm{O}-\mathrm{H} \cdots \mathrm{O}$ and $\mathrm{N}-\mathrm{H} \cdots \mathrm{O}$ hydrogen bonds together with weak $\mathrm{C}-\mathrm{H} \cdots \mathrm{Cl}$ and $\mathrm{C}-\mathrm{H} \cdots \mathrm{O}$ hydrogen bonds $(\mathrm{C} 8-$ $\mathrm{H} 8 \cdots \mathrm{Cl} 1^{\mathrm{iii}}$ and $\mathrm{C} 8-\mathrm{H} 8 \cdots \mathrm{O} 1^{\mathrm{iii}}$; Table 1 ), forming a layer parallel to the $a b$ plane (Fig. 4). In the layer, the chloranilic acid rings are stacked along the $b$ axis through a $\pi-\pi$ interaction [centroid-centroid distance $=3.6851$ (7) $\AA$ and interplanar spacing $=3.2118(4) \AA]$. The pyridine rings are also

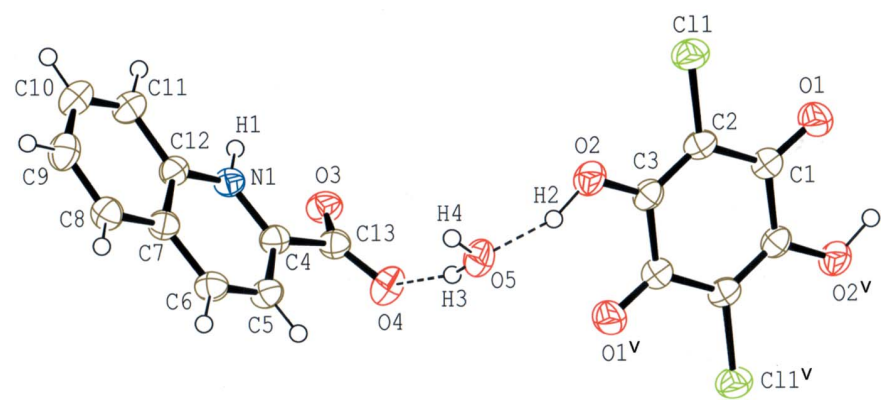

Figure 3

The molecular structure of compound (II), showing the atom-numbering scheme. Displacement ellipsoids of non-H atoms are drawn at the $50 \%$ probability level and $\mathrm{H}$ atoms are drawn as small spheres of arbitrary radii. $\mathrm{O}-\mathrm{H} \cdots \mathrm{O}$ hydrogen bonds are shown as dashed lines. [Symmetry code: $(\mathrm{v})-x,-y,-z$.]
Table 2

Hydrogen-bond geometry $\left(\AA,^{\circ}\right)$ for (II).

\begin{tabular}{lllll}
\hline$D-\mathrm{H} \cdots A$ & $D-\mathrm{H}$ & $\mathrm{H} \cdots A$ & $D \cdots A$ & $D-\mathrm{H} \cdots A$ \\
\hline $\mathrm{N} 1-\mathrm{H} 1 \cdots \mathrm{O} 3^{\mathrm{i}}$ & $0.88(2)$ & $1.91(2)$ & $2.7724(17)$ & $167(2)$ \\
$\mathrm{O} 2-\mathrm{H} 2 \cdots \mathrm{O} 5$ & $0.98(3)$ & $1.59(3)$ & $2.5092(17)$ & $155(3)$ \\
$\mathrm{O} 5-\mathrm{H} 3 \cdots \mathrm{O} 4$ & $0.82(2)$ & $2.01(2)$ & $2.8072(19)$ & $164(2)$ \\
$\mathrm{O}^{\mathrm{O}}-\mathrm{H} 4 \cdots \mathrm{O} 4^{\text {ii }}$ & $0.85(2)$ & $1.82(2)$ & $2.6632(19)$ & $171(2)$ \\
$\mathrm{C} 6-\mathrm{H} 6 \cdots 1^{\text {iii }}$ & 0.95 & 2.54 & $3.392(2)$ & 150 \\
${\text { C6-H6 } \cdots 5^{\text {iv }}}$ & 0.95 & 2.47 & $3.211(2)$ & 134 \\
\hline
\end{tabular}

Symmetry codes: (i) $-x,-y+1,-z+1$; (ii) $x+1, y, z$; (iii) $x+1, y, z+1$; (iv) $-x+1,-y,-z+1$.

stacked along the $b$ axis through a $\pi-\pi$ interaction [centroidcentroid distance $=3.6851(7) \AA$ and interplanar spacing = $3.4787(5) \AA]$. Between the layers, a short $\mathrm{Cl} \cdots \mathrm{Cl}$ contact is observed $\left[\mathrm{Cl} 1 \cdots \mathrm{Cl} 1^{\mathrm{v}}=3.3717(5) \AA\right.$; symmetry code: $(\mathrm{v})$ $\left.-x+1, y-\frac{1}{2},-z+\frac{1}{2}\right]$.

In the crystal of (II), two adjacent 2-carboxyquinoline molecules, which are related by an inversion centre, form a head-to-tail dimer via a pair of $\mathrm{N}-\mathrm{H} \cdots \mathrm{O}$ hydrogen bonds $\left(\mathrm{N}-\mathrm{H} 1 \cdots \mathrm{O} 3^{\mathrm{i}}\right.$; symmetry code as in Table 2$)$. The dimers are stacked in a column along the $a$ axis through a weak $\pi-\pi$ interaction between the N1/C4-C7/C12 and C7-C12 rings with a centroid-centroid distance of 3.9184 (10) $\AA$. The water molecule links the stacked base molecules related by translation along a via $\mathrm{O}-\mathrm{H} \cdots \mathrm{O}$ hydrogen bonds [O5- $\mathrm{H} 3 \cdots \mathrm{O} 4$ and $\mathrm{O} 5-\mathrm{H} 4 \cdots \mathrm{O} 4{ }^{\mathrm{ii}}$; Table 2] and also links the acid molecule and the two base molecules via $\mathrm{O}-\mathrm{H} \cdots \mathrm{O}$ hydrogen bonds, forming a layer structure parallel to (011) as shown in Fig. 5. No significant short contact between the acid molecules in the layer is observed. Between the layers, a bifurcated $\mathrm{C}-\mathrm{H} \cdots(\mathrm{O}$, $\mathrm{O})$ hydrogen bond $\left(\mathrm{C} 6-\mathrm{H} 6 \cdots \mathrm{O} 1^{\mathrm{iii}}\right.$ and $\mathrm{C} 6-\mathrm{H} 6 \cdots \mathrm{O} 5^{\mathrm{iv}}$; Table 2) is observed, through which the 2-carboxyquinoline molecule is weakly linked with the chloranilic acid and water molecules.

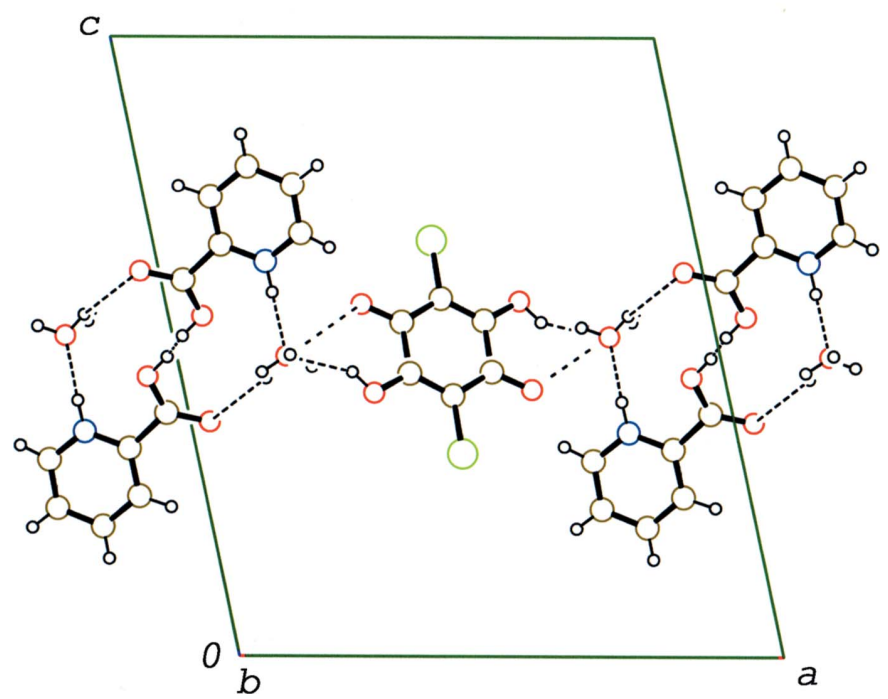

Figure 4

A packing diagram of compound (I) viewed along the $b$ axis, showing the layer structure. $\mathrm{O}-\mathrm{H} \cdots \mathrm{O}$ and $\mathrm{N}-\mathrm{H} \cdots \mathrm{O}$ hydrogen bonds are shown as dashed lines. 
Table 3

Experimental details.

(I)

Crystal data

Chemical formula

$M_{\mathrm{r}}$

Crystal system, space group

Temperature $(\mathrm{K})$

$a, b, c(\AA)$

$\alpha, \beta, \gamma\left({ }^{\circ}\right)$

$V\left(\AA^{3}\right)$

Z

Radiation type

$\mu\left(\mathrm{mm}^{-1}\right)$

Crystal size (mm)

Data collection

Diffractometer

Absorption correction

$T_{\min }, T_{\max }$

No. of measured, independent and

observed $[I>2 \sigma(I)]$ reflections

$R_{\text {int }}$

$(\sin \theta / \lambda)_{\max }\left(\AA^{-1}\right)$

Refinement

$R\left[F^{2}>2 \sigma\left(F^{2}\right)\right], w R\left(F^{2}\right), S$

No. of reflections

No. of parameters

No. of restraints

$\mathrm{H}$-atom treatment

$\Delta \rho_{\max }, \Delta \rho_{\min }\left(\mathrm{e} \AA^{-3}\right)$

0.021

0.704

(II)
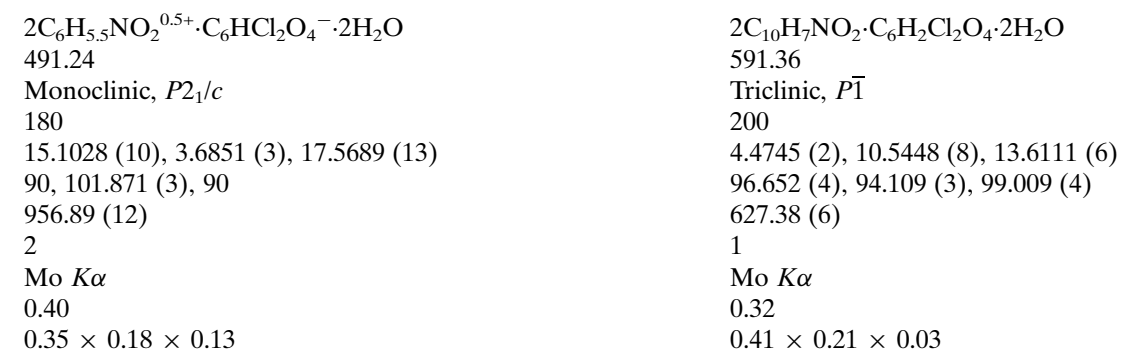

Rigaku R-AXIS RAPIDII

Numerical (NUMABS; Higashi, 1999)

$0.896,0.949$

$17752,2789,2537$

Rigaku R-AXIS RAPIDII

Numerical (NUMABS; Higashi, 1999)

$0.925,0.990$

$12358,3666,2755$

0.122

0.704

$\begin{array}{ll}0.030,0.079,1.11 & 0.052,0.149,1.01 \\ 2789 & 3666 \\ 176 & 197 \\ 8 & 2 \\ \text { H atoms treated by a mixture of independent } & \text { H atoms treated by a mixture of independent } \\ \quad \text { and constrained refinement } & \text { and constrained refinement } \\ 0.54,-0.24 & 0.52,-0.45\end{array}$

Computer programs: RAPID-AUTO (Rigaku, 2006), SHELXS97 (Sheldrick, 2008), SHELXL2016 (Sheldrick, 2015), ORTEP-3 for Windows (Farrugia, 2012), CrystalStructure (Rigaku, 2010) and PLATON (Spek, 2015).

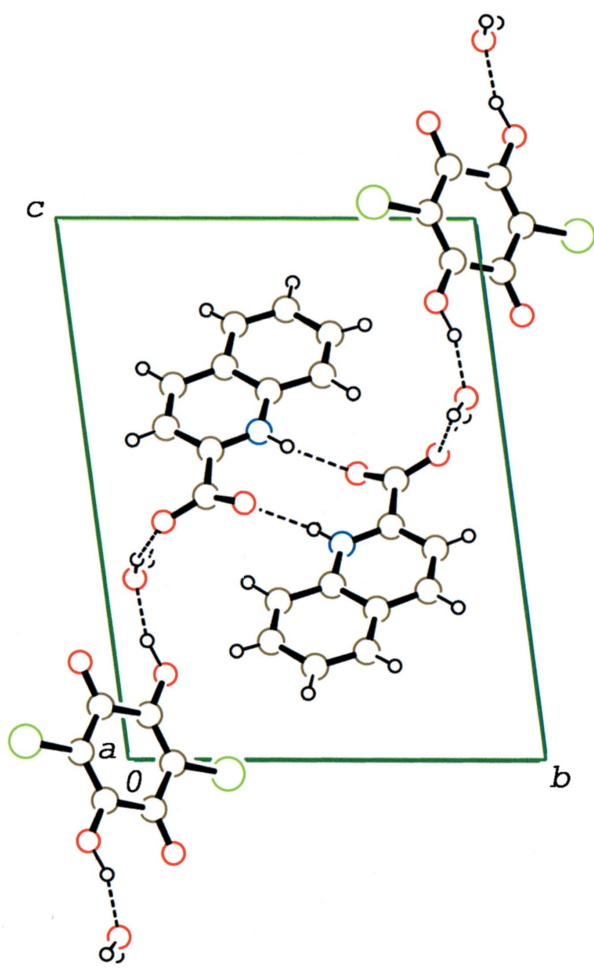

Figure 5

A packing diagram of compound (II) viewed along the $a$ axis, showing the layer structure formed by $\mathrm{O}-\mathrm{H} \cdots \mathrm{O}$ and $\mathrm{N}-\mathrm{H} \cdots \mathrm{O}$ hydrogen bonds (dashed lines).

\section{Database survey}

A search of the Cambridge Structural Database (Version 5.38, last update May 2017; Groom et al., 2016) for organic cocrystals of pyridinium-2-carboxylate (twitterionic form) gave six structures. For organic co-crystals of quinolinium-2carboxylate (twitterionic form), eight structures were found.

\section{Synthesis and crystallization}

Single crystals of compound (I) were obtained by slow evaporation of an acetonitrile solution $(200 \mathrm{ml})$ of chloranilic acid $(250 \mathrm{mg})$ with 2-carboxypridine $(310 \mathrm{mg})$ at room temperature. Single crystals of compound (II) were obtained by slow evaporation from a methanol solution $(150 \mathrm{ml})$ of chloranilic acid $(310 \mathrm{mg})$ with 2-carboxyquinoline $(520 \mathrm{mg})$ at room temperature.

\section{Refinement}

Crystal data, data collection and structure refinement details are summarized in Table 3. The water molecule in compound (I) was found to be disordered over two sites in a differenceFourier map. The occupancies were refined to 0.52 (2) and 0.48 (2) and then they were fixed at 0.5 . The $\mathrm{H}$ atom in the carboxy group of the base molecule was also found in a 
difference-Fourier map to be disordered between the adjacent carboxy groups, which are related by an inversion centre, and the occupancy was set to be 0.5 . Since the N-bound $\mathrm{H}$ atom refined reasonably with an occupancy of 1 , the occupancy of the $\mathrm{H}$ atom of the acid molecule was set to be 0.5 to balance the total charge of the compound. All other $\mathrm{H}$ atoms were found in a difference-Fourier map. The $\mathrm{N}$-bound $\mathrm{H}$ atom was refined freely, while the positions of $\mathrm{O}$-bound $\mathrm{H}$ atoms were refined, with $\mathrm{O}-\mathrm{H}=0.84$ (2) $\AA$ and $U_{\text {iso }}(\mathrm{H})=1.5 U_{\text {eq }}(\mathrm{O})$. For the water $\mathrm{H}$ atoms, distant restraints of $\mathrm{H} \cdots \mathrm{H}=1.37$ (4) $\AA$ were also applied. C-bound $\mathrm{H}$ atoms were positioned geometrically $(\mathrm{C}-\mathrm{H}=0.95 \AA)$ and were treated as riding with $U_{\text {iso }}(\mathrm{H})=1.2 U_{\text {eq }}(\mathrm{C})$.

All $\mathrm{H}$ atoms in compound (II) were found in a differenceFourier map. The $\mathrm{O}$ - and $\mathrm{N}$-bound $\mathrm{H}$ atoms in the acid and base molecules were refined freely. The water $\mathrm{H}$ atoms were refined with $\mathrm{O}-\mathrm{H}=0.84$ (2) $\AA$. C-bound $\mathrm{H}$ atoms were positioned geometrically $(\mathrm{C}-\mathrm{H}=0.95 \AA)$ and were treated as riding with $U_{\text {iso }}(\mathrm{H})=1.2 U_{\text {eq }}(\mathrm{C})$.

\section{References}

Asaji, T., Seliger, J., Žagar, V. \& Ishida, H. (2010). Magn. Reson. Chem. 48, 531-536.
Farrugia, L. J. (2012). J. Appl. Cryst. 45, 849-854.

Gotoh, K., Nagoshi, H. \& Ishida, H. (2009). Acta Cryst. E65, o614.

Gotoh, K., Tabuchi, Y., Akashi, H. \& Ishida, H. (2006). Acta Cryst. E62, o4420-04421.

Groom, C. R., Bruno, I. J., Lightfoot, M. P. \& Ward, S. C. (2016). Acta Cryst. B72, 171-179.

Higashi, T. (1999). NUMABS. Rigaku Corporation, Tokyo, Japan.

Marfo-Owusu, E. \& Thompson, A. L. (2014). X-ray Struct. Anal. Online, 30, 55-56.

Marfo-Owusu, E. \& Thompson, A. L. (2016). X-ray Struct. Anal. Online, 32, 49-50.

Molčanov, K. \& Kojić-Prodić, B. (2010). CrystEngComm, 12, $925-$ 939.

Rigaku (2006). RAPID-AUTO. Rigaku Corporation, Tokyo, Japan.

Rigaku (2010). CrystalStructure. Rigaku Corporation, Tokyo, Japan.

Seliger, J., Žagar, V., Gotoh, K., Ishida, H., Konnai, A., Amino, D. \& Asaji, T. (2009). Phys. Chem. Chem. Phys. 11, 2281-2286.

Sheldrick, G. M. (2008). Acta Cryst. A64, 112-122.

Sheldrick, G. M. (2015). Acta Cryst. C71, 3-8.

Spek, A. L. (2015). Acta Cryst. C71, 9-18.

Tabuchi, Y., Takahashi, A., Gotoh, K., Akashi, H. \& Ishida, H. (2005). Acta Cryst. E61, o4215-o4217.

Wang, B.-Q., Yan, H.-B., Huang, Z.-Q., Zhang, Y.-H. \& Sun, J. (2015). Acta Cryst. C71, 247-251.

Zaman, Md. B., Udachin, K. A. \& Ripmeester, J. A. (2004). Cryst. Growth Des. 4, 585-589. 


\section{supporting information}

Acta Cryst. (2017). E73, 1840-1844 [https://doi.org/10.1107/S2056989017015997]

\section{Crystal structures of two 1:2 dihydrate compounds of chloranilic acid with 2- carboxypyridine and 2-carboxyquinoline}

\section{Kazuma Gotoh and Hiroyuki Ishida}

\section{Computing details}

For both structures, data collection: RAPID-AUTO (Rigaku, 2006); cell refinement: RAPID-AUTO (Rigaku, 2006); data reduction: RAPID-AUTO (Rigaku, 2006); program(s) used to solve structure: SHELXS97 (Sheldrick, 2008); program(s) used to refine structure: SHELXL2016 (Sheldrick, 2015); molecular graphics: ORTEP-3 for Windows (Farrugia, 2012); software used to prepare material for publication: CrystalStructure (Rigaku, 2010) and PLATON (Spek, 2015).

Bis(2-carboxypyridinium) chloranilate dihydrate-bis(pyridinium-2-carboxylate) chloranilic acid dihydrate (1/1)

(I)

\section{Crystal data}

$2 \mathrm{C}_{6} \mathrm{H}_{5.5} \mathrm{NO}_{2} 0.5+\cdot \mathrm{C}_{6} \mathrm{HCl}_{2} \mathrm{O}_{4}{ }^{-} \cdot 2 \mathrm{H}_{2} \mathrm{O}$

$F(000)=504.00$

$M_{r}=491.24$

Monoclinic, $P 2{ }_{1} / c$

$a=15.1028(10) \AA$

$b=3.6851(3) \AA$

$c=17.5689(13) \AA$

$\beta=101.871(3)^{\circ}$

$V=956.89(12) \AA^{3}$

$D_{\mathrm{x}}=1.705 \mathrm{Mg} \mathrm{m}^{-3}$

Mo $K \alpha$ radiation, $\lambda=0.71075 \AA$

$Z=2$

Cell parameters from 15838 reflections

$\theta=3.2-30.0^{\circ}$

$\mu=0.40 \mathrm{~mm}^{-1}$

$T=180 \mathrm{~K}$

Block, brown

$0.35 \times 0.18 \times 0.13 \mathrm{~mm}$

\section{Data collection}

Rigaku R-AXIS RAPIDII diffractometer

Detector resolution: 10.000 pixels $\mathrm{mm}^{-1}$

$\omega$ scans

Absorption correction: numerical

(NUMABS; Higashi, 1999)

$T_{\min }=0.896, T_{\max }=0.949$

17752 measured reflections

2789 independent reflections 2537 reflections with $I>2 \sigma(I)$

$R_{\text {int }}=0.021$

$\theta_{\max }=30.0^{\circ}, \theta_{\min }=3.3^{\circ}$

$h=-21 \rightarrow 21$

$k=-5 \rightarrow 4$

$l=-24 \rightarrow 24$

\section{Refinement}

Refinement on $F^{2}$

$R\left[F^{2}>2 \sigma\left(F^{2}\right)\right]=0.030$

$w R\left(F^{2}\right)=0.079$

$S=1.11$

2789 reflections

176 parameters

8 restraints
Primary atom site location: structure-invariant direct methods

Secondary atom site location: difference Fourier map

Hydrogen site location: difference Fourier map

$\mathrm{H}$ atoms treated by a mixture of independent and constrained refinement 
$w=1 /\left[\sigma^{2}\left(F_{\mathrm{o}}^{2}\right)+(0.0445 P)^{2}+0.3065 P\right]$

where $P=\left(F_{\mathrm{o}}^{2}+2 F_{\mathrm{c}}^{2}\right) / 3$

$(\Delta / \sigma)_{\max }=0.001$

$$
\Delta \rho_{\max }=0.54 \mathrm{e} \AA^{-3}
$$

Special details

Geometry. All esds (except the esd in the dihedral angle between two 1.s. planes) are estimated using the full covariance matrix. The cell esds are taken into account individually in the estimation of esds in distances, angles and torsion angles; correlations between esds in cell parameters are only used when they are defined by crystal symmetry. An approximate (isotropic) treatment of cell esds is used for estimating esds involving 1.s. planes.

Fractional atomic coordinates and isotropic or equivalent isotropic displacement parameters $\left(\AA^{2}\right)$

\begin{tabular}{llllll}
\hline & $x$ & $y$ & $z$ & $U_{\text {iso }} * / U_{\text {eq }}$ & Occ. $(<1)$ \\
\hline C11 & $0.48813(2)$ & $0.21752(7)$ & $0.32764(2)$ & $0.01728(8)$ & \\
O1 & $0.64061(5)$ & $0.6016(2)$ & $0.43110(4)$ & $0.02004(16)$ & \\
O2 & $0.34886(5)$ & $0.1370(2)$ & $0.42588(4)$ & $0.02038(17)$ & 0.5 \\
H2 & $0.317(2)$ & $0.137(9)$ & $0.4593(16)$ & $0.031^{*}$ & \\
O3 & $0.06134(7)$ & $0.3759(3)$ & $0.54909(5)$ & $0.0313(2)$ & 0.5 \\
H3 & $0.018(2)$ & $0.445(12)$ & $0.516(2)$ & $0.047^{*}$ & \\
O4 & $-0.03494(6)$ & $0.1653(3)$ & $0.62162(5)$ & $0.0303(2)$ & 0.5 \\
O5A & $0.19794(11)$ & $0.1042(7)$ & $0.48308(10)$ & $0.0265(4)$ & 0.5 \\
H9A & $0.1547(18)$ & $0.038(11)$ & $0.4493(18)$ & $0.040^{*}$ & 0.5 \\
H10A & $0.2453(15)$ & $0.043(11)$ & $0.4682(18)$ & $0.040^{*}$ & 0.5 \\
O5B & $0.20132(11)$ & $-0.1191(6)$ & $0.48529(9)$ & $0.0199(3)$ & \\
H9B & $0.1501(15)$ & $-0.090(9)$ & $0.4587(19)$ & $0.030^{*}$ & \\
H10B & $0.209(2)$ & $-0.347(5)$ & $0.4873(19)$ & $0.030^{*}$ & \\
N1 & $0.19857(6)$ & $0.0209(3)$ & $0.63805(5)$ & $0.01977(18)$ & \\
C1 & $0.57374(6)$ & $0.5511(3)$ & $0.45987(5)$ & $0.01438(18)$ & \\
C2 & $0.49294(7)$ & $0.3688(3)$ & $0.42158(5)$ & $0.01476(18)$ & \\
C3 & $0.42138(6)$ & $0.3090(3)$ & $0.45734(6)$ & $0.01574(19)$ & \\
C4 & $0.12231(7)$ & $0.0793(3)$ & $0.66495(6)$ & $0.01658(19)$ & \\
C5 & $0.12193(7)$ & $0.0166(3)$ & $0.74206(6)$ & $0.0198(2)$ & \\
H5 & 0.068573 & 0.056090 & 0.761605 & $0.024 *$ & \\
C6 & $0.20078(8)$ & $-0.1057(3)$ & $0.79117(6)$ & $0.0226(2)$ & \\
H6 & 0.201710 & -0.146932 & 0.844701 & $0.027^{*}$ & \\
C7 & $0.27772(7)$ & $-0.1668(3)$ & $0.76174(7)$ & $0.0226(2)$ & \\
H7 & 0.331694 & -0.251819 & 0.794609 & $0.027^{*}$ & \\
C8 & $0.27478(7)$ & $-0.1023(3)$ & $0.68384(7)$ & $0.0236(2)$ & \\
H8 & 0.326860 & -0.145258 & 0.662656 & $0.028^{*}$ & \\
C9 & $0.04024(8)$ & $0.2152(3)$ & $0.60747(6)$ & $0.0210(2)$ & \\
H1 & $0.1993(12)$ & $0.049(5)$ & $0.5876(10)$ & $0.039(4)^{*}$ & \\
& & & & & \\
\end{tabular}

Atomic displacement parameters $\left(\AA^{2}\right)$

\begin{tabular}{lllllll}
\hline & $U^{11}$ & $U^{22}$ & $U^{33}$ & $U^{12}$ & $U^{13}$ & $U^{23}$ \\
\hline C11 & $0.01956(13)$ & $0.02040(13)$ & $0.01184(12)$ & $-0.00159(8)$ & $0.00314(8)$ & $-0.00202(8)$ \\
O1 & $0.0170(3)$ & $0.0273(4)$ & $0.0174(3)$ & $-0.0029(3)$ & $0.0071(3)$ & $-0.0028(3)$ \\
O2 & $0.0159(3)$ & $0.0286(4)$ & $0.0168(3)$ & $-0.0068(3)$ & $0.0037(3)$ & $-0.0038(3)$ \\
O3 & $0.0340(5)$ & $0.0363(5)$ & $0.0200(4)$ & $-0.0003(4)$ & $-0.0028(3)$ & $0.0107(4)$
\end{tabular}


supporting information

$\begin{array}{lllllll}\text { O4 } & 0.0212(4) & 0.0433(5) & 0.0243(4) & 0.0042(4) & -0.0003(3) & 0.0001(4) \\ \text { O5A } & 0.0152(8) & 0.0467(13) & 0.0185(8) & -0.0033(8) & 0.0052(6) & -0.0080(8) \\ \text { O5B } & 0.0160(7) & 0.0243(9) & 0.0188(7) & -0.0013(7) & 0.0028(5) & -0.0010(7) \\ \text { N1 } & 0.0231(4) & 0.0215(4) & 0.0160(4) & 0.0002(4) & 0.0071(3) & -0.0002(3) \\ \text { C1 } & 0.0148(4) & 0.0154(4) & 0.0132(4) & 0.0008(3) & 0.0035(3) & 0.0012(3) \\ \text { C2 } & 0.0171(4) & 0.0174(4) & 0.0100(4) & -0.0015(3) & 0.0033(3) & -0.0007(3) \\ \text { C3 } & 0.0147(4) & 0.0186(5) & 0.0137(4) & 0.0012(4) & 0.0025(3) & 0.0021(3) \\ \text { C4 } & 0.0177(4) & 0.0163(4) & 0.0151(4) & -0.0002(4) & 0.0022(3) & 0.0005(3) \\ \text { C5 } & 0.0183(4) & 0.0251(5) & 0.0166(4) & 0.0003(4) & 0.0052(3) & 0.0026(4) \\ \text { C6 } & 0.0236(5) & 0.0271(6) & 0.0163(4) & -0.0015(4) & 0.0018(4) & 0.0051(4) \\ \text { C7 } & 0.0185(5) & 0.0209(5) & 0.0261(5) & 0.0010(4) & -0.0009(4) & 0.0032(4) \\ \text { C8 } & 0.0197(5) & 0.0245(5) & 0.0281(5) & 0.0025(4) & 0.0083(4) & -0.0013(4) \\ \text { C9 } & 0.0241(5) & 0.0207(5) & 0.0156(4) & 0.0022(4) & -0.0017(4) & -0.0013(4) \\ \end{array}$

Geometric parameters $\left(\AA,{ }^{o}\right)$

\begin{tabular}{|c|c|c|c|}
\hline $\mathrm{C} 11-\mathrm{C} 2$ & $1.7293(9)$ & $\mathrm{N} 1-\mathrm{H} 1$ & $0.895(18)$ \\
\hline $\mathrm{O} 1-\mathrm{C} 1$ & $1.2330(11)$ & $\mathrm{C} 1-\mathrm{C} 2$ & $1.4335(13)$ \\
\hline $\mathrm{O} 2-\mathrm{C} 3$ & $1.2875(12)$ & $\mathrm{C} 1-\mathrm{C} 3^{\mathrm{i}}$ & $1.5307(13)$ \\
\hline $\mathrm{O} 2-\mathrm{H} 2$ & $0.830(18)$ & $\mathrm{C} 2-\mathrm{C} 3$ & $1.3751(13)$ \\
\hline $\mathrm{O} 3-\mathrm{C} 9$ & $1.2801(14)$ & $\mathrm{C} 4-\mathrm{C} 5$ & $1.3754(13)$ \\
\hline $\mathrm{O} 3-\mathrm{H} 3$ & $0.814(17)$ & $\mathrm{C} 4-\mathrm{C} 9$ & $1.5139(14)$ \\
\hline $\mathrm{O} 4-\mathrm{C} 9$ & $1.2250(15)$ & $\mathrm{C} 5-\mathrm{C} 6$ & $1.3939(15)$ \\
\hline $\mathrm{O} 5 \mathrm{~A}-\mathrm{H} 9 \mathrm{~A}$ & $0.824(18)$ & $\mathrm{C} 5-\mathrm{H} 5$ & 0.9500 \\
\hline $\mathrm{O} 5 \mathrm{~A}-\mathrm{H} 10 \mathrm{~A}$ & $0.841(18)$ & $\mathrm{C} 6-\mathrm{C} 7$ & $1.3840(16)$ \\
\hline $\mathrm{O} 5 \mathrm{~B}-\mathrm{H} 9 \mathrm{~B}$ & $0.824(17)$ & $\mathrm{C} 6-\mathrm{H} 6$ & 0.9500 \\
\hline $\mathrm{O} 5 \mathrm{~B}-\mathrm{H} 10 \mathrm{~B}$ & $0.849(17)$ & $\mathrm{C} 7-\mathrm{C} 8$ & $1.3808(16)$ \\
\hline $\mathrm{N} 1-\mathrm{C} 8$ & $1.3406(14)$ & $\mathrm{C} 7-\mathrm{H} 7$ & 0.9500 \\
\hline $\mathrm{N} 1-\mathrm{C} 4$ & $1.3492(13)$ & $\mathrm{C} 8-\mathrm{H} 8$ & 0.9500 \\
\hline $\mathrm{C} 3-\mathrm{O} 2-\mathrm{H} 2$ & $105(2)$ & $\mathrm{N} 1-\mathrm{C} 4-\mathrm{C} 9$ & $117.38(9)$ \\
\hline $\mathrm{C} 9-\mathrm{O} 3-\mathrm{H} 3$ & $115(3)$ & $\mathrm{C} 5-\mathrm{C} 4-\mathrm{C} 9$ & $122.95(9)$ \\
\hline $\mathrm{H} 9 \mathrm{~A}-\mathrm{O} 5 \mathrm{~A}-\mathrm{H} 10 \mathrm{~A}$ & $107(3)$ & $\mathrm{C} 4-\mathrm{C} 5-\mathrm{C} 6$ & $119.20(9)$ \\
\hline $\mathrm{H} 9 \mathrm{~B}-\mathrm{O} 5 \mathrm{~B}-\mathrm{H} 10 \mathrm{~B}$ & $105(3)$ & $\mathrm{C} 4-\mathrm{C} 5-\mathrm{H} 5$ & 120.4 \\
\hline $\mathrm{C} 8-\mathrm{N} 1-\mathrm{C} 4$ & $122.23(9)$ & $\mathrm{C} 6-\mathrm{C} 5-\mathrm{H} 5$ & 120.4 \\
\hline $\mathrm{C} 8-\mathrm{N} 1-\mathrm{H} 1$ & $116.7(11)$ & $\mathrm{C} 7-\mathrm{C} 6-\mathrm{C} 5$ & $119.83(10)$ \\
\hline $\mathrm{C} 4-\mathrm{N} 1-\mathrm{H} 1$ & $120.9(11)$ & $\mathrm{C} 7-\mathrm{C} 6-\mathrm{H} 6$ & 120.1 \\
\hline $\mathrm{O} 1-\mathrm{C} 1-\mathrm{C} 2$ & $124.63(9)$ & $\mathrm{C} 5-\mathrm{C} 6-\mathrm{H} 6$ & 120.1 \\
\hline $\mathrm{O} 1-\mathrm{C} 1-\mathrm{C} 3^{\mathrm{i}}$ & $117.08(9)$ & $\mathrm{C} 8-\mathrm{C} 7-\mathrm{C} 6$ & $118.95(10)$ \\
\hline $\mathrm{C} 2-\mathrm{C} 1-\mathrm{C} 3^{\mathrm{i}}$ & $118.29(8)$ & $\mathrm{C} 8-\mathrm{C} 7-\mathrm{H} 7$ & 120.5 \\
\hline $\mathrm{C} 3-\mathrm{C} 2-\mathrm{C} 1$ & $122.26(8)$ & $\mathrm{C} 6-\mathrm{C} 7-\mathrm{H} 7$ & 120.5 \\
\hline $\mathrm{C} 3-\mathrm{C} 2-\mathrm{C} 11$ & $120.12(8)$ & $\mathrm{N} 1-\mathrm{C} 8-\mathrm{C} 7$ & $120.11(10)$ \\
\hline $\mathrm{C} 1-\mathrm{C} 2-\mathrm{C} 11$ & $117.62(7)$ & $\mathrm{N} 1-\mathrm{C} 8-\mathrm{H} 8$ & 119.9 \\
\hline $\mathrm{O} 2-\mathrm{C} 3-\mathrm{C} 2$ & $124.19(9)$ & $\mathrm{C} 7-\mathrm{C} 8-\mathrm{H} 8$ & 119.9 \\
\hline $\mathrm{O} 2-\mathrm{C} 3-\mathrm{C}^{\mathrm{i}}$ & $116.38(8)$ & $\mathrm{O} 4-\mathrm{C} 9-\mathrm{O} 3$ & $128.74(11)$ \\
\hline $\mathrm{C} 2-\mathrm{C} 3-\mathrm{C}^{\mathrm{i}}$ & $119.43(8)$ & $\mathrm{O} 4-\mathrm{C} 9-\mathrm{C} 4$ & $118.74(10)$ \\
\hline $\mathrm{N} 1-\mathrm{C} 4-\mathrm{C} 5$ & $119.67(9)$ & $\mathrm{O} 3-\mathrm{C} 9-\mathrm{C} 4$ & $112.51(10)$ \\
\hline
\end{tabular}




$\begin{array}{ll}\mathrm{O} 1-\mathrm{C} 1-\mathrm{C} 2-\mathrm{C} 3 & 177.79(10) \\ \mathrm{C} 3-\mathrm{C} 1-\mathrm{C} 2-\mathrm{C} 3 & -1.60(16) \\ \mathrm{O} 1-\mathrm{C} 1-\mathrm{C} 2-\mathrm{C} 11 & -1.39(14) \\ \mathrm{C} 3-\mathrm{C} 1-\mathrm{C} 2-\mathrm{C} 11 & 179.22(7) \\ \mathrm{C} 1-\mathrm{C} 2-\mathrm{C} 3-\mathrm{O} 2 & -177.81(10) \\ \mathrm{C} 11-\mathrm{C} 2-\mathrm{C} 3-\mathrm{O} 2 & 1.35(15) \\ \mathrm{C} 1-\mathrm{C} 2-\mathrm{C} 3-\mathrm{C}^{\mathrm{i}} & 1.61(16) \\ \mathrm{C} 11-\mathrm{C} 2-\mathrm{C} 3-\mathrm{C} 1^{\mathrm{i}} & -179.22(7) \\ \mathrm{C} 8-\mathrm{N} 1-\mathrm{C} 4-\mathrm{C} 5 & 0.93(17) \\ \mathrm{C} 8-\mathrm{N} 1-\mathrm{C} 4-\mathrm{C} 9 & -179.41(10)\end{array}$

$0.18(17)$

$-179.46(10)$

$-0.86(17)$

$0.46(18)$

$-1.34(17)$

$0.62(18)$

$157.55(11)$

$-22.81(16)$

$-23.17(14)$

$156.48(11)$

Symmetry code: (i) $-x+1,-y+1,-z+1$.

Hydrogen-bond geometry $\left(\AA,{ }^{\circ}\right)$

\begin{tabular}{lllll}
\hline$D-\mathrm{H} \cdots A$ & $D-\mathrm{H}$ & $\mathrm{H} \cdots A$ & $D \cdots A$ & $D-\mathrm{H} \cdots A$ \\
\hline $\mathrm{N} 1-\mathrm{H} 1 \cdots \mathrm{O} 5 A$ & $0.895(17)$ & $1.843(17)$ & $2.7380(19)$ & $179(2)$ \\
$\mathrm{N} 1-\mathrm{H} 1 \cdots \mathrm{O} 5 B$ & $0.895(17)$ & $1.907(17)$ & $2.7417(18)$ & $154.4(17)$ \\
$\mathrm{O} 2-\mathrm{H} 2 \cdots \mathrm{O} 5 B$ & $0.83(3)$ & $2.12(3)$ & $2.8111(19)$ & $141(3)$ \\
$\mathrm{O} 3-\mathrm{H} 3 \cdots \mathrm{O} 3{ }^{\mathrm{ii}}$ & $0.82(3)$ & $1.62(4)$ & $2.4352(14)$ & $174(4)$ \\
$\mathrm{O} 5 A-\mathrm{H} 9 A \cdots \mathrm{O} 4{ }^{i i i}$ & $0.82(3)$ & $2.11(3)$ & $2.927(2)$ & $170(3)$ \\
$\mathrm{O} 5 B-\mathrm{H} 9 B \cdots \mathrm{O} 4^{i i i}$ & $0.82(3)$ & $2.02(3)$ & $2.8159(19)$ & $162(3)$ \\
$\mathrm{O} 5 A-\mathrm{H} 10 A \cdots \mathrm{O} 2$ & $0.84(3)$ & $1.90(3)$ & $2.6762(19)$ & $154(4)$ \\
$\mathrm{O} 5 B-\mathrm{H} 10 B \cdots \mathrm{O} 1^{\text {iv }}$ & $0.85(2)$ & $2.60(3)$ & $3.095(2)$ & $119(2)$ \\
$\mathrm{C} 8-\mathrm{H} 8 \cdots \mathrm{Cl}^{1 i}$ & 0.95 & 2.78 & $3.6524(12)$ & 154 \\
$\mathrm{C} 8-\mathrm{H} 8 \cdots{ }^{i v}$ & 0.95 & 2.47 & $3.1871(14)$ & 132
\end{tabular}

Symmetry codes: (ii) $-x,-y+1,-z+1$; (iii) $-x,-y,-z+1$; (iv) $-x+1,-y,-z+1$.

chloranilic acid-2-carboxyquinoline (1/2) dihydrate (II)

\section{Crystal data}

$2 \mathrm{C}_{10} \mathrm{H}_{7} \mathrm{NO}_{2} \cdot \mathrm{C}_{6} \mathrm{H}_{2} \mathrm{Cl}_{2} \mathrm{O}_{4} \cdot 2 \mathrm{H}_{2} \mathrm{O}$

$M_{r}=591.36$

Triclinic, $P \overline{1}$

Hall symbol: -P 1

$a=4.4745(2) \AA$

$b=10.5448(8) \AA$

$c=13.6111(6) \AA$

$\alpha=96.652(4)^{\circ}$

$\beta=94.109(3)^{\circ}$

$\gamma=99.009(4)^{\circ}$

$V=627.38(6) \AA^{3}$

\section{Data collection}

Rigaku R-AXIS RAPIDII diffractometer

Detector resolution: 10.000 pixels $\mathrm{mm}^{-1}$

$\omega$ scans

Absorption correction: numerical

(NUMABS; Higashi, 1999)

$T_{\min }=0.925, T_{\max }=0.990$

12358 measured reflections
$Z=1$

$F(000)=304.00$

$D_{\mathrm{x}}=1.565 \mathrm{Mg} \mathrm{m}^{-3}$

Mo $K \alpha$ radiation, $\lambda=0.71075 \AA$

Cell parameters from 9714 reflections

$\theta=3.0-30.1^{\circ}$

$\mu=0.32 \mathrm{~mm}^{-1}$

$T=200 \mathrm{~K}$

Platelet, brown

$0.41 \times 0.21 \times 0.03 \mathrm{~mm}$

3666 independent reflections

2755 reflections with $I>2 \sigma(I)$

$R_{\text {int }}=0.122$

$\theta_{\max }=30.0^{\circ}, \theta_{\min }=3.0^{\circ}$

$h=-6 \rightarrow 6$

$k=-14 \rightarrow 14$

$l=-19 \rightarrow 19$ 


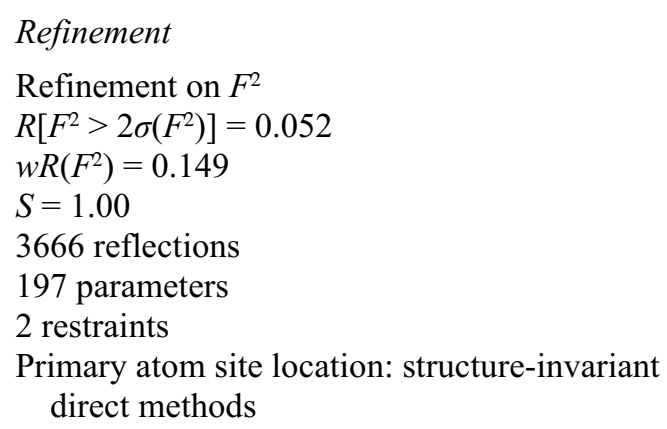

Secondary atom site location: difference Fourier map

Hydrogen site location: inferred from neighbouring sites

$\mathrm{H}$ atoms treated by a mixture of independent and constrained refinement

$w=1 /\left[\sigma^{2}\left(F_{0}^{2}\right)+(0.0879 P)^{2}\right]$ where $P=\left(F_{\mathrm{o}}^{2}+2 F_{\mathrm{c}}^{2}\right) / 3$

$(\Delta / \sigma)_{\max }<0.001$

$\Delta \rho_{\max }=0.52 \mathrm{e}^{-3}$

Special details

Geometry. All esds (except the esd in the dihedral angle between two 1.s. planes) are estimated using the full covariance matrix. The cell esds are taken into account individually in the estimation of esds in distances, angles and torsion angles; correlations between esds in cell parameters are only used when they are defined by crystal symmetry. An approximate (isotropic) treatment of cell esds is used for estimating esds involving l.s. planes.

Fractional atomic coordinates and isotropic or equivalent isotropic displacement parameters $\left(\hat{A}^{2}\right)$

\begin{tabular}{lllll}
\hline & $x$ & $y$ & $z$ & $U_{\text {iso }} * / U_{\text {eq }}$ \\
\hline C11 & $0.49390(9)$ & $0.23664(4)$ & $-0.02842(3)$ & $0.04300(16)$ \\
O1 & $0.0319(3)$ & $0.08081(12)$ & $-0.17653(8)$ & $0.0426(3)$ \\
O2 & $0.4150(3)$ & $0.11710(11)$ & $0.15510(9)$ & $0.0391(3)$ \\
O3 & $-0.1769(3)$ & $0.36216(11)$ & $0.47121(9)$ & $0.0372(3)$ \\
O4 & $-0.0712(3)$ & $0.16160(11)$ & $0.43604(9)$ & $0.0425(3)$ \\
O5 & $0.3938(3)$ & $0.07471(12)$ & $0.33231(9)$ & $0.0406(3)$ \\
N1 & $0.3233(3)$ & $0.41970(12)$ & $0.60390(9)$ & $0.0298(3)$ \\
C1 & $0.0207(3)$ & $0.04635(14)$ & $-0.09469(11)$ & $0.0317(3)$ \\
C2 & $0.2276(3)$ & $0.10762(14)$ & $-0.00985(11)$ & $0.0320(3)$ \\
C3 & $0.2197(3)$ & $0.06514(14)$ & $0.07971(11)$ & $0.0313(3)$ \\
C4 & $0.2151(3)$ & $0.29703(14)$ & $0.56909(11)$ & $0.0305(3)$ \\
C5 & $0.3274(3)$ & $0.19702(15)$ & $0.61140(11)$ & $0.0336(3)$ \\
H5 & 0.250293 & 0.109195 & 0.586437 & $0.040^{*}$ \\
C6 & $0.5493(4)$ & $0.22680(15)$ & $0.68906(12)$ & $0.0342(3)$ \\
H6 & 0.622423 & 0.159293 & 0.719133 & $0.041^{*}$ \\
C7 & $0.6694(3)$ & $0.35659(14)$ & $0.72449(11)$ & $0.0312(3)$ \\
C8 & $0.9050(4)$ & $0.39332(16)$ & $0.80217(12)$ & $0.0363(3)$ \\
H8 & 0.985438 & 0.328869 & 0.834065 & $0.044^{*}$ \\
C9 & $1.0170(4)$ & $0.52094(18)$ & $0.83147(13)$ & $0.0411(4)$ \\
H9 & 1.175836 & 0.545100 & 0.883608 & $0.049^{*}$ \\
C10 & $0.8980(4)$ & $0.61764(17)$ & $0.78463(13)$ & $0.0418(4)$ \\
H10 & 0.980263 & 0.706066 & 0.805678 & $0.050^{*}$ \\
C11 & $0.6677(4)$ & $0.58722(15)$ & $0.70983(13)$ & $0.0372(3)$ \\
H11 & 0.587483 & 0.653070 & 0.679646 & $0.045^{*}$ \\
C12 & $0.5532(3)$ & $0.45558(14)$ & $0.67897(10)$ & $0.0294(3)$ \\
C13 & $-0.0333(3)$ & $0.27243(14)$ & $0.48397(11)$ & $0.0315(3)$ \\
H1 & $0.252(5)$ & $0.481(2)$ & $0.5760(17)$ & $0.055(6)^{*}$ \\
H2 & $0.359(7)$ & $0.083(3)$ & $0.216(2)$ & $0.092(10)^{*}$ \\
& & & &
\end{tabular}




\begin{tabular}{lllll}
$\mathrm{H} 3$ & $0.255(4)$ & $0.086(2)$ & $0.3667(15)$ & $0.054(6)^{*}$ \\
$\mathrm{H} 4$ & $0.560(4)$ & $0.111(2)$ & $0.3651(17)$ & $0.063(7)^{*}$ \\
\hline
\end{tabular}

Atomic displacement parameters $\left(\AA^{2}\right)$

\begin{tabular}{lllllll}
\hline & $U^{11}$ & $U^{22}$ & $U^{33}$ & $U^{12}$ & $U^{13}$ & $U^{23}$ \\
\hline C11 & $0.0402(2)$ & $0.0380(2)$ & $0.0465(2)$ & $-0.00570(16)$ & $-0.00095(18)$ & $0.00725(17)$ \\
O1 & $0.0451(7)$ & $0.0452(7)$ & $0.0354(6)$ & $0.0008(5)$ & $-0.0026(5)$ & $0.0093(5)$ \\
O2 & $0.0388(6)$ & $0.0380(6)$ & $0.0359(6)$ & $-0.0005(5)$ & $-0.0079(5)$ & $0.0012(4)$ \\
O3 & $0.0376(6)$ & $0.0319(6)$ & $0.0427(6)$ & $0.0068(4)$ & $-0.0017(5)$ & $0.0089(4)$ \\
O4 & $0.0403(6)$ & $0.0368(6)$ & $0.0471(6)$ & $0.0091(5)$ & $-0.0071(5)$ & $-0.0052(5)$ \\
O5 & $0.0400(7)$ & $0.0419(7)$ & $0.0357(6)$ & $0.0047(5)$ & $-0.0066(5)$ & $-0.0029(5)$ \\
N1 & $0.0302(6)$ & $0.0269(6)$ & $0.0326(6)$ & $0.0053(5)$ & $0.0020(5)$ & $0.0055(5)$ \\
C1 & $0.0292(7)$ & $0.0304(7)$ & $0.0353(7)$ & $0.0061(5)$ & $-0.0004(6)$ & $0.0039(6)$ \\
C2 & $0.0287(7)$ & $0.0274(7)$ & $0.0384(7)$ & $0.0022(5)$ & $-0.0010(6)$ & $0.0032(5)$ \\
C3 & $0.0298(7)$ & $0.0278(7)$ & $0.0354(7)$ & $0.0064(5)$ & $-0.0016(6)$ & $0.0007(5)$ \\
C4 & $0.0297(7)$ & $0.0291(7)$ & $0.0330(7)$ & $0.0046(5)$ & $0.0044(6)$ & $0.0045(5)$ \\
C5 & $0.0347(7)$ & $0.0273(7)$ & $0.0384(7)$ & $0.0043(5)$ & $0.0005(6)$ & $0.0051(5)$ \\
C6 & $0.0357(7)$ & $0.0301(7)$ & $0.0378(7)$ & $0.0059(6)$ & $0.0020(6)$ & $0.0089(6)$ \\
C7 & $0.0299(7)$ & $0.0315(7)$ & $0.0325(7)$ & $0.0047(5)$ & $0.0039(6)$ & $0.0054(5)$ \\
C8 & $0.0347(7)$ & $0.0388(8)$ & $0.0353(7)$ & $0.0063(6)$ & $0.0011(6)$ & $0.0060(6)$ \\
C9 & $0.0358(8)$ & $0.0453(9)$ & $0.0382(8)$ & $0.0033(7)$ & $-0.0036(7)$ & $-0.0030(7)$ \\
C10 & $0.0402(8)$ & $0.0329(8)$ & $0.0484(9)$ & $0.0026(6)$ & $0.0006(7)$ & $-0.0046(7)$ \\
C11 & $0.0376(8)$ & $0.0289(7)$ & $0.0443(8)$ & $0.0061(6)$ & $0.0027(7)$ & $0.0014(6)$ \\
C12 & $0.0281(6)$ & $0.0287(7)$ & $0.0311(6)$ & $0.0044(5)$ & $0.0042(6)$ & $0.0021(5)$ \\
C13 & $0.0293(7)$ & $0.0311(7)$ & $0.0339(7)$ & $0.0037(5)$ & $0.0024(6)$ & $0.0058(5)$ \\
& & & & & &
\end{tabular}

Geometric parameters $\left(\hat{A},{ }^{\circ}\right)$

\begin{tabular}{llll}
\hline $\mathrm{C} 11-\mathrm{C} 2$ & $1.7174(15)$ & $\mathrm{C} 4-\mathrm{C} 13$ & $1.517(2)$ \\
$\mathrm{O} 1-\mathrm{C} 1$ & $1.2130(18)$ & $\mathrm{C} 5-\mathrm{C} 6$ & $1.370(2)$ \\
$\mathrm{O} 2-\mathrm{C} 3$ & $1.3070(17)$ & $\mathrm{C} 5-\mathrm{H} 5$ & 0.9500 \\
$\mathrm{O} 2-\mathrm{H} 2$ & $0.98(3)$ & $\mathrm{C} 6-\mathrm{C} 7$ & $1.405(2)$ \\
$\mathrm{O} 3-\mathrm{C} 13$ & $1.2453(18)$ & $\mathrm{C} 6-\mathrm{H} 6$ & 0.9500 \\
$\mathrm{O} 4-\mathrm{C} 13$ & $1.2512(18)$ & $\mathrm{C} 7-\mathrm{C} 8$ & $1.413(2)$ \\
$\mathrm{O} 5-\mathrm{H} 3$ & $0.821(16)$ & $\mathrm{C} 7-\mathrm{C} 12$ & $1.420(2)$ \\
$\mathrm{O} 5-\mathrm{H} 4$ & $0.848(16)$ & $\mathrm{C} 8-\mathrm{C} 9$ & $1.363(2)$ \\
$\mathrm{N} 1-\mathrm{C} 4$ & $1.3270(19)$ & $\mathrm{C} 8-\mathrm{H} 8$ & 0.9500 \\
$\mathrm{~N} 1-\mathrm{C} 12$ & $1.3722(19)$ & $\mathrm{C} 9-\mathrm{C} 10$ & $1.415(3)$ \\
$\mathrm{N} 1-\mathrm{H} 1$ & $0.88(2)$ & $\mathrm{C} 9-\mathrm{H} 9$ & 0.9500 \\
$\mathrm{C} 1-\mathrm{C} 2$ & $1.447(2)$ & $\mathrm{C} 10-\mathrm{C} 11$ & $1.368(2)$ \\
$\mathrm{C} 1-\mathrm{C} 3$ & $\mathrm{C} 10-\mathrm{H} 10$ & 0.9500 \\
$\mathrm{C} 2-\mathrm{C} 3$ & $1.507(2)$ & $\mathrm{C} 11-\mathrm{C} 12$ & $1.406(2)$ \\
$\mathrm{C} 4-\mathrm{C} 5$ & $1.348(2)$ & $\mathrm{C} 11-\mathrm{H} 11$ & 0.9500 \\
& $1.401(2)$ & & 119.8 \\
$\mathrm{C} 3-\mathrm{O} 2-\mathrm{H} 2$ & & $\mathrm{C} 7-\mathrm{C} 6-\mathrm{H} 6$ & $123.01(14)$ \\
$\mathrm{H} 3-\mathrm{O} 5-\mathrm{H} 4$ & $111.3(18)$ & $\mathrm{C} 6-\mathrm{C} 7-\mathrm{C} 8$ & $118.61(13)$ \\
$\mathrm{C} 4-\mathrm{N} 1-\mathrm{C} 12$ & $108(2)$ & $\mathrm{C} 6-\mathrm{C} 7-\mathrm{C} 12$ &
\end{tabular}




\begin{tabular}{|c|c|c|c|}
\hline $\mathrm{C} 4-\mathrm{N} 1-\mathrm{H} 1$ & $119.1(16)$ & $\mathrm{C} 8-\mathrm{C} 7-\mathrm{C} 12$ & $118.37(14)$ \\
\hline $\mathrm{C} 12-\mathrm{N} 1-\mathrm{H} 1$ & $117.9(16)$ & $\mathrm{C} 9-\mathrm{C} 8-\mathrm{C} 7$ & $120.18(15)$ \\
\hline $\mathrm{O} 1-\mathrm{C} 1-\mathrm{C} 2$ & $123.09(14)$ & $\mathrm{C} 9-\mathrm{C} 8-\mathrm{H} 8$ & 119.9 \\
\hline $\mathrm{O} 1-\mathrm{C} 1-\mathrm{C} 3^{\mathrm{i}}$ & $118.94(13)$ & $\mathrm{C} 7-\mathrm{C} 8-\mathrm{H} 8$ & 119.9 \\
\hline $\mathrm{C} 2-\mathrm{C} 1-\mathrm{C} 3^{\mathrm{i}}$ & $117.97(13)$ & $\mathrm{C} 8-\mathrm{C} 9-\mathrm{C} 10$ & $120.32(15)$ \\
\hline $\mathrm{C} 3-\mathrm{C} 2-\mathrm{C} 1$ & $122.39(13)$ & $\mathrm{C} 8-\mathrm{C} 9-\mathrm{H} 9$ & 119.8 \\
\hline $\mathrm{C} 3-\mathrm{C} 2-\mathrm{C} 11$ & $120.62(11)$ & $\mathrm{C} 10-\mathrm{C} 9-\mathrm{H} 9$ & 119.8 \\
\hline $\mathrm{C} 1-\mathrm{C} 2-\mathrm{C} 11$ & $116.98(11)$ & $\mathrm{C} 11-\mathrm{C} 10-\mathrm{C} 9$ & $121.78(15)$ \\
\hline $\mathrm{O} 2-\mathrm{C} 3-\mathrm{C} 2$ & $122.30(14)$ & $\mathrm{C} 11-\mathrm{C} 10-\mathrm{H} 10$ & 119.1 \\
\hline $\mathrm{O} 2-\mathrm{C} 3-\mathrm{C}^{\mathrm{i}}$ & $118.13(13)$ & $\mathrm{C} 9-\mathrm{C} 10-\mathrm{H} 10$ & 119.1 \\
\hline $\mathrm{C} 2-\mathrm{C} 3-\mathrm{C} 1^{\mathrm{i}}$ & $119.57(12)$ & $\mathrm{C} 10-\mathrm{C} 11-\mathrm{C} 12$ & $118.00(15)$ \\
\hline $\mathrm{N} 1-\mathrm{C} 4-\mathrm{C} 5$ & $120.22(13)$ & $\mathrm{C} 10-\mathrm{C} 11-\mathrm{H} 11$ & 121.0 \\
\hline $\mathrm{N} 1-\mathrm{C} 4-\mathrm{C} 13$ & $116.94(13)$ & $\mathrm{C} 12-\mathrm{C} 11-\mathrm{H} 11$ & 121.0 \\
\hline $\mathrm{C} 5-\mathrm{C} 4-\mathrm{C} 13$ & $122.84(13)$ & $\mathrm{N} 1-\mathrm{C} 12-\mathrm{C} 11$ & $120.39(13)$ \\
\hline $\mathrm{C} 6-\mathrm{C} 5-\mathrm{C} 4$ & $119.50(14)$ & $\mathrm{N} 1-\mathrm{C} 12-\mathrm{C} 7$ & $118.27(13)$ \\
\hline $\mathrm{C} 6-\mathrm{C} 5-\mathrm{H} 5$ & 120.2 & $\mathrm{C} 11-\mathrm{C} 12-\mathrm{C} 7$ & $121.34(14)$ \\
\hline $\mathrm{C} 4-\mathrm{C} 5-\mathrm{H} 5$ & 120.2 & $\mathrm{O} 3-\mathrm{C} 13-\mathrm{O} 4$ & $128.04(14)$ \\
\hline $\mathrm{C} 5-\mathrm{C} 6-\mathrm{C} 7$ & $120.39(14)$ & $\mathrm{O} 3-\mathrm{C} 13-\mathrm{C} 4$ & $117.19(13)$ \\
\hline $\mathrm{C} 5-\mathrm{C} 6-\mathrm{H} 6$ & 119.8 & $\mathrm{O} 4-\mathrm{C} 13-\mathrm{C} 4$ & $114.76(13)$ \\
\hline $\mathrm{O} 1-\mathrm{C} 1-\mathrm{C} 2-\mathrm{C} 3$ & $-177.43(15)$ & $\mathrm{C} 12-\mathrm{C} 7-\mathrm{C} 8-\mathrm{C} 9$ & $-0.4(2)$ \\
\hline $\mathrm{C} 3-\mathrm{C} 1-\mathrm{C} 2-\mathrm{C} 3$ & $2.8(2)$ & $\mathrm{C} 7-\mathrm{C} 8-\mathrm{C} 9-\mathrm{C} 10$ & $0.2(3)$ \\
\hline $\mathrm{O} 1-\mathrm{C} 1-\mathrm{C} 2-\mathrm{C} 11$ & $1.2(2)$ & $\mathrm{C} 8-\mathrm{C} 9-\mathrm{C} 10-\mathrm{C} 11$ & $0.5(3)$ \\
\hline $\mathrm{C} 33^{\mathrm{i}}-\mathrm{C} 1-\mathrm{C} 2-\mathrm{C} 11$ & $-178.60(10)$ & $\mathrm{C} 9-\mathrm{C} 10-\mathrm{C} 11-\mathrm{C} 12$ & $-1.0(3)$ \\
\hline $\mathrm{C} 1-\mathrm{C} 2-\mathrm{C} 3-\mathrm{O} 2$ & $177.00(13)$ & $\mathrm{C} 4-\mathrm{N} 1-\mathrm{C} 12-\mathrm{C} 11$ & $177.18(14)$ \\
\hline $\mathrm{C} 11-\mathrm{C} 2-\mathrm{C} 3-\mathrm{O} 2$ & $-1.6(2)$ & $\mathrm{C} 4-\mathrm{N} 1-\mathrm{C} 12-\mathrm{C} 7$ & $-3.2(2)$ \\
\hline $\mathrm{C} 1-\mathrm{C} 2-\mathrm{C} 3-\mathrm{C}^{\mathrm{i}}$ & $-2.8(2)$ & $\mathrm{C} 10-\mathrm{C} 11-\mathrm{C} 12-\mathrm{N} 1$ & $-179.65(14)$ \\
\hline $\mathrm{C} 11-\mathrm{C} 2-\mathrm{C} 3-\mathrm{C}^{\mathrm{i}}$ & $178.61(10)$ & $\mathrm{C} 10-\mathrm{C} 11-\mathrm{C} 12-\mathrm{C} 7$ & $0.7(2)$ \\
\hline $\mathrm{C} 12-\mathrm{N} 1-\mathrm{C} 4-\mathrm{C} 5$ & $2.4(2)$ & $\mathrm{C} 6-\mathrm{C} 7-\mathrm{C} 12-\mathrm{N} 1$ & $1.5(2)$ \\
\hline $\mathrm{C} 12-\mathrm{N} 1-\mathrm{C} 4-\mathrm{C} 13$ & $-178.34(12)$ & $\mathrm{C} 8-\mathrm{C} 7-\mathrm{C} 12-\mathrm{N} 1$ & $-179.66(13)$ \\
\hline $\mathrm{N} 1-\mathrm{C} 4-\mathrm{C} 5-\mathrm{C} 6$ & $0.1(2)$ & $\mathrm{C} 6-\mathrm{C} 7-\mathrm{C} 12-\mathrm{C} 11$ & $-178.84(14)$ \\
\hline $\mathrm{C} 13-\mathrm{C} 4-\mathrm{C} 5-\mathrm{C} 6$ & $-179.12(14)$ & $\mathrm{C} 8-\mathrm{C} 7-\mathrm{C} 12-\mathrm{C} 11$ & $0.0(2)$ \\
\hline $\mathrm{C} 4-\mathrm{C} 5-\mathrm{C} 6-\mathrm{C} 7$ & $-1.6(2)$ & $\mathrm{N} 1-\mathrm{C} 4-\mathrm{C} 13-\mathrm{O} 3$ & $-19.8(2)$ \\
\hline $\mathrm{C} 5-\mathrm{C} 6-\mathrm{C} 7-\mathrm{C} 8$ & $-177.95(14)$ & $\mathrm{C} 5-\mathrm{C} 4-\mathrm{C} 13-\mathrm{O} 3$ & $159.47(14)$ \\
\hline $\mathrm{C} 5-\mathrm{C} 6-\mathrm{C} 7-\mathrm{C} 12$ & $0.8(2)$ & $\mathrm{N} 1-\mathrm{C} 4-\mathrm{C} 13-\mathrm{O} 4$ & $161.50(14)$ \\
\hline $\mathrm{C} 6-\mathrm{C} 7-\mathrm{C} 8-\mathrm{C} 9$ & $178.31(16)$ & $\mathrm{C} 5-\mathrm{C} 4-\mathrm{C} 13-\mathrm{O} 4$ & $-19.3(2)$ \\
\hline
\end{tabular}

Symmetry code: (i) $-x,-y,-z$.

Hydrogen-bond geometry $\left(A,{ }^{\circ}\right)$

\begin{tabular}{lllll}
\hline$D-\mathrm{H} \cdots A$ & $D-\mathrm{H}$ & $\mathrm{H} \cdots A$ & $D \cdots A$ & $D-\mathrm{H}^{\cdots} A$ \\
\hline $\mathrm{N} 1-\mathrm{H} 1 \cdots \mathrm{O} 33^{\mathrm{ii}}$ & $0.88(2)$ & $1.91(2)$ & $2.7724(17)$ & $167(2)$ \\
$\mathrm{O} 2-\mathrm{H} 2 \cdots \mathrm{O} 5$ & $0.98(3)$ & $1.59(3)$ & $2.5092(17)$ & $155(3)$ \\
$\mathrm{O} 5-\mathrm{H} 3 \cdots \mathrm{O} 4$ & $0.82(2)$ & $2.01(2)$ & $2.8072(19)$ & $164(2)$ \\
$\mathrm{O} 5-\mathrm{H} 4 \cdots \mathrm{O} 44^{\mathrm{iii}}$ & $0.85(2)$ & $1.82(2)$ & $2.6632(19)$ & $171(2)$
\end{tabular}


supporting information

$\begin{array}{lllll}\mathrm{C} 6-\mathrm{H} 6 \cdots \mathrm{O} 1^{\text {iv }} & 0.95 & 2.54 & 3.392(2) & 150 \\ \mathrm{C} 6-\mathrm{H} 6 \cdots 5^{\mathrm{v}} & 0.95 & 2.47 & 3.211(2) & 134\end{array}$

Symmetry codes: (ii) $-x,-y+1,-z+1$; (iii) $x+1, y, z$; (iv) $x+1, y, z+1$; (v) $-x+1,-y,-z+1$. 